\title{
Splenic Arteriogastric Fistula Resulting from Adrenocortical Carcinoma: A Rare Cause of Massive Upper Gastrointestinal Hemorrhage
}

\author{
Elias J. Dayoub ${ }^{1,2}$, Kyung J. Cho ${ }^{3^{*}}$ \\ ${ }^{1}$ University of Michigan Medical School, Ann Arbor, USA \\ ${ }^{2}$ John F. Kennedy School of Government, Harvard University, Cambridge, USA \\ ${ }^{3}$ Department of Radiology, Division of Vascular \& Interventional Radiology, \\ University of Michigan Health System, Ann Arbor, USA \\ Email: *kyungcho@med.umich.edu
}

Received October 8, 2013; revised November 8, 2013; accepted November 15, 2013

Copyright (c) 2013 Elias J. Dayoub, Kyung J. Cho. This is an open access article distributed under the Creative Commons Attribution License, which permits unrestricted use, distribution, and reproduction in any medium, provided the original work is properly cited. In accordance of the Creative Commons Attribution License all Copyrights (C) 2013 are reserved for SCIRP and the owner of the intellectual property Elias J. Dayoub, Kyung J. Cho. All Copyright (C) 2013 are guarded by law and by SCIRP as a guardian.

\begin{abstract}
Splenic arteriogastric fistula is a rare cause of upper gastrointestinal (GI) hemorrhage, only reported a handful of times in the literature. Herein, we present a case of a 49-year-old woman with metastatic adrenocortical carcincoma who developed a fistula between the splenic artery and gastric lumen as a result of local invasion of her primary tumor. This fistula led to several episodes of massive upper GI bleeding. Selective splenic artery embolization was successful in ceasing the GI bleed; however, the intervention was not timely enough for the patient to survive the hemorrhage. We outline several clinical and imaging findings to assist physicians with earlier detection of splenic arteriogastric fistulas, and advocate prophylactic selective splenic artery embolization when this rare but highly fatal entity is discovered.
\end{abstract}

Keywords: Splenic Artery; Fistula; Pseudoaneurysm; Adrenocortical Carcinoma; Embolization

\section{Introduction}

Splenic arteriogastric fistula is a rare cause of gastrointestinal hemorrhage. Reports in the literature have associated this often-fatal condition with suture plication of a giant gastric ulcer, sleeve gastrectomy, severe peptic ulcer disease, and splenic artery aneurysms fistulizing in the setting of pancreatic pseudocysts [1-6]. To our knowledge, we are reporting the first case of splenic arteriogastric fistula caused by adrenocortical carcinoma or any other solid tumor. Further, we discuss clinical and imaging findings which aid in identifying splenic arteriogastric fistulas and propose prophylactic selective splenic artery embolization as appropriate management when this rare but highly fatal condition is suspected.

\section{Case Summary}

In 2011, a 49-year-old woman presented with signs of hypercortisolism secondary to adrenocortical carcinoma. Initial CT imaging revealed a $16.3 \times 7.7 \mathrm{~cm}$ mass in her left adrenal gland, along with multiple metastases present in her liver and lungs. She underwent multiple cycles of chemotherapy and radiation therapy of the adrenal mass and liver metastases, but continued to have progression of her disease.

Two years later, she presented with an episode of large volume hematemesis. Urgent esophagogastroduodenoscopy was performed on admission, revealing blood in the gastric cardia with a large blood clot obscuring the exact source of bleeding. A Sengstaken-Blakemore tube was placed, successfully tamponading the bleed. CT angiography was performed and no active bleeding source was found, though this initial study did find delayed filling of the splenic artery that was attributed to local compression from the Blakemore tube. The following day, the Blakemore tube was removed and a repeat endoscopy was performed. No active bleeding was noted this time; however, the clot in the gastric cardia continued to obscure the underlying mucosa. No varices, ulcer, or tumor was seen. Nonetheless, tumor invasion into the posterior 
wall of the stomach was the presumed etiology of the GI bleed. The patient remained stable for days with no signs of GI bleeding and was subsequently discharged.

She returned three days later with complaints of severe epigastric pain. A CT abdomen was performed which revealed no acute bleeding (Figure 1(A)). The splenic vessels appeared widely patent proximally and distally, but the mid-portion was poorly visualized with abutment of the primary tumor. On the second day of hospitalizetion, the patient began having multiple episodes of hematemesis. Urgent endoscopy was performed, revealing no active bleeding and again the large clot in the gastric cardia was seen. Interventional radiology was subsequently consulted to perform catheter-based angiography.

Arteriograms of the abdominal aorta and visceral ves- sels were performed. Significant tumor blush was present in the liver and near the celiac trunk. Focal stenosis was seen in the left renal artery and mid-splenic artery, consistent with extrinsic compression or invasion of the primary tumor. A subtle billowing of the mid-splenic artery was appreciated as a possible pseudoaneurysm, though the finding was considered indeterminate at the time. Otherwise, no extravasation or arterial findings amenable to endovascular treatment were noted.

The following morning, the patient had hematemesis and signs of hemorrhagic shock. She was resuscitated with isotonic saline, blood products, and maximum dosages of norepinephrine (Levophed) and urgently transferred to the angiography suite. A celiac arteriogram was subsequently performed which showed significant vasospasm with severe extravasation from the mid-splenic
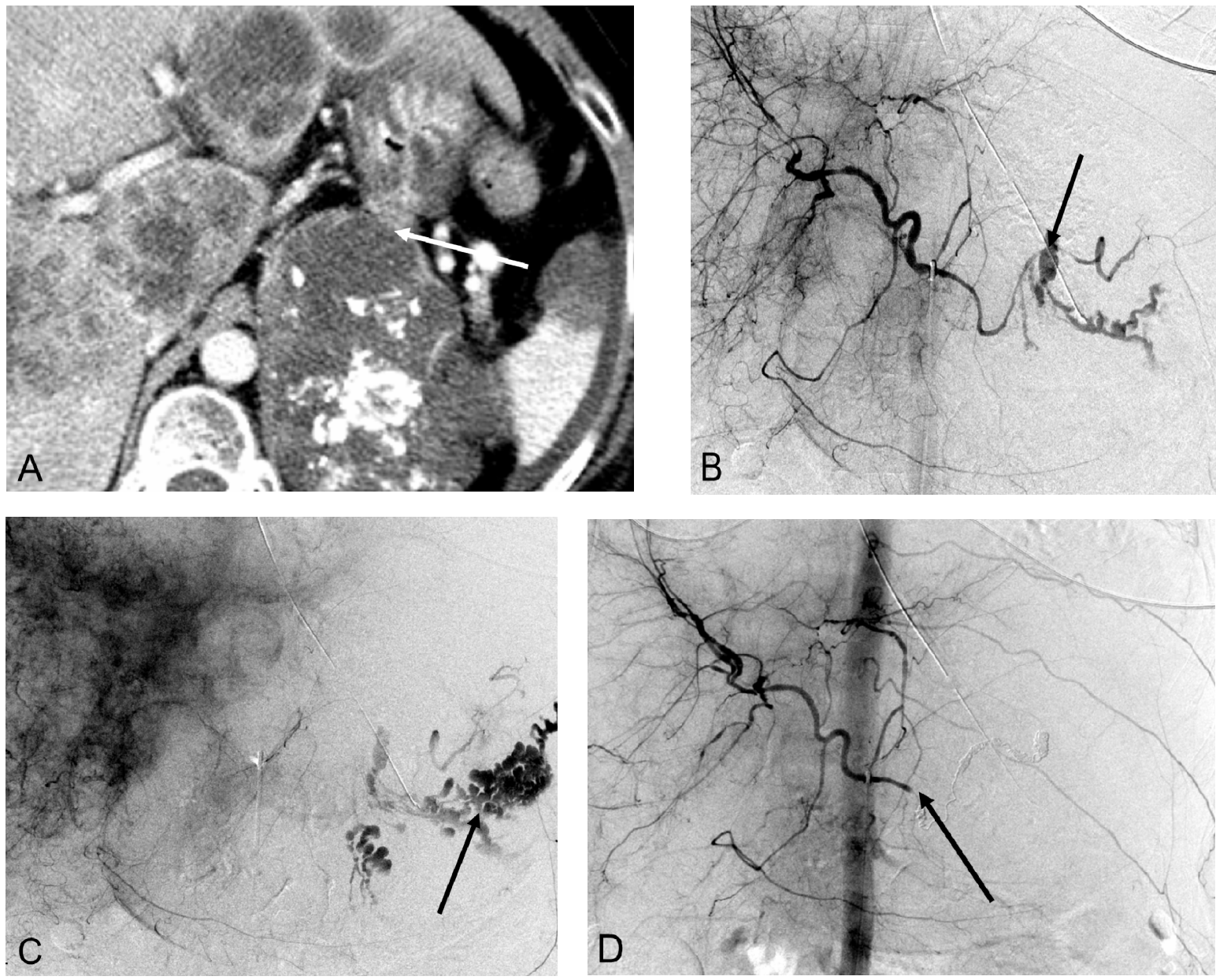

Figure 1. (A) CT scan section after injection of intravenous contrast showing a large enhancing adrenocortical carcinoma abutting the posterior gastric wall (arrow); (B) Celiac digital subtraction angiogram (DSA) shows massive extravasation of contrast medium from narrowed mid-portion of the splenic artery into the lumen of the stomach (arrow). The splenic, gastroduodenal and left gastric arteries, and their branches demonstrate severe vasoconstriction but to a lesser extent as a result of hepatic artery "escape" responding to decreased portal blood flow; (C) Late arterial phase of the celiac DSA. The extravasated contrast medium collected in the lumen of the stomach outlining its mucosa (arrow); (D) Celiac angiogram following selective embolization of the splenic artery with coils and Gelfoam pledget shows occlusion of the splenic artery (arrow). No contrast medium extravasated. 
artery, with a more distinguishable pseudoaneurysm rupturing directly into the gastric lumen (Figures 1(B) and (C)). The site of splenic artery injury corresponded to the encased portion of the artery between the left adrenal mass and posterior wall of the stomach. A microcatheter was placed across the site of splenic artery rupture, then splenic artery embolization was performed with multiple coils including $6 \mathrm{~mm}$ in diameter Tornado microcoils (Cook, Bloomington, IN, USA), a $6 \mathrm{~mm}$ in diameter 0.018" Nester coils (Cook, Bloomington, IN, USA), and Gelfoam pledgets (Pfizer, New York, NY, USA). Contrast was gently hand injected through the microcatheter, confirming stasis of flow. CO2 was also injected, which confirmed stasis of flow into the splenic artery. No significant collateral supply demonstrated extravasation. Finally, completion celiac arteriogram also demonstrated lack of antegrade flow in the splenic artery (Figure 1(D)).

The patient was transferred back to the intensive care unit and received continued hemodynamic support; however, she continued to have worsening hypotension and tachycardia. The patient became increasingly unresponsive, vital signs continued to worsen, and she passed away within 12 hours of the splenic artery embolization.

\section{Discussion}

Management of splenic arteriogastric fistulas in previous case reports has included embolization and splenectomy with splenic artery ligation [1-6]. Despite these efforts, these cases had a high rate of mortality, likely due to interventions occurring after patients already suffered significant gastrointestinal hemorrhage. Similarly, we did not intervene with splenic artery embolization until the patient became hemodynamically unstable. Though we successfully performed selective mid-splenic artery embolization of the pseudoaneurysm responsible for the bleed, the patient died hours after the procedure. Several opportunities were presented where earlier intervention would have likely resulted in longer survival for the patient. We recommend prophylactic splenic artery embolization when moderate suspicion for splenic arteriogastric fistula as a source of gastrointestinal hemorrhage exists.

Several findings in this case should raise suspicion for splenic arteriogastric fistula as the source of gastrointestinal bleeding. The first diagnostic and therapeutic intervention typically employed in acute upper GI bleeding is endoscopy [7]. Endoscopy was not only helpful in ruling out the more common causes of GI bleeding, but also localized the bleed to the posterior wall of the stomach. CT imaging and digital subtraction angiography (DSA) showed tumor encasing a portion of the splenic artery and tumor apposing the posterior wall of the stomach, consistent with the site of bleeding on endoscopy. DSA also revealed focal stenosis of the mid-portion of the splenic artery, with the presence of a small dilatation within the stenosis, vaguely suggestive of a pseudoaneurysm. Close examination for pseudoaneurysms should be performed in this presentation, given that its presence is a clear indication for catheter-based embolization or thrombin injection via CT guidance [8].

Even in the absence of a clear pseudoaneurysm, a combination of the findings above should warrant serious consideration of prophylactic selective splenic artery embolization. Precise embolization of the pseudoaneurysm or area of focal stenosis is necessary to preserve collateral flow to the spleen via the gastric, omental, and pancreatic vessels. If performed successfully, complications are uncommon and typically limited to post-embolization syndrome, though splenic infarction, infection and splenic abscess are still possible [9]. Nonetheless, the risk of these potential complications is eclipsed by the overwhelming benefit of avoiding the morbidity and mortality from an actively hemorrhaging splenic arteriogastric fistula.

\section{REFERENCES}

[1] L. Rebibo, D. Fuks, C. Blot, B. Robert, P. O. Boulet, A. Dhahri, P. Verhaeghe and J. M. Regimbeau, "Gastrointestinal Bleeding Complication of Gastric Fistula after Sleeve Gastrectomy: Consider Pseudoaneurysms," Surgical Endoscopy, Vol. 27, No. 8, 2013, pp. 2849-2855. http://dx.doi.org/10.1007/s00464-013-2833-7

[2] S. S. Morse, B. N. Siskind, N. R. Horowitz and E. B. Strauss, "Splenic Arteriogastric Fistula from Plication of a Gastric Ulcer. Therapeutic Embolization,” Journal of Clinical Gastroenterology, Vol. 9, No. 4, 1987, pp. 480482.

http://dx.doi.org/10.1097/00004836-198708000-00026

[3] E. Moreno González, A. García Ocaña, J. Hebrero Sanmartin, M. Moreno Azcoita, A. Belda Serna, and J. L. Rodriguez Agullo, "Fistula between the Splenic Artery and the Gastric Cavity as an Exceptional Complication of Peptic Ulcer. Report of a Case,” Revista Espanola de las Enfermedades del Aparto Digestivo, Vol. 53, No. 5, 1978, pp. 543-554.

[4] T. Cioppa, A. De Stefano, D. Marrelli, A. Neri, S. Rossi, G. De Marco, E. Pinto and F. Roviello, "Pseudoaneurysm of the Splenic Artery Fistulized in the Stomach and Associated to a Pancreatic Pseudocyst: Case Report," Minerva Chirurgica, Vol. 61, No. 3, 2006, pp. 261-264.

[5] T. Nakazawa, M. Kida, S. Kokubu, S. Tanabe, A. Shibuya, K. Matsunaga and K. Saigenji, "Massive Gastrointestinal Hemorrhage from Pseudocyst with Fistula and Splenic Artery Aneurysm Rupture,” Endoscopy, Vol. 31, No. 3, 1999, p. S23.

[6] D. A. Toroptsev, A. V. Anisimov and F. S. Zhizhin, "Gastrointestinal Hemorrhage Caused by Rupture of Aneurysm of the Splenic Artery into the Stomach," Vestnik Khirurgii Imeni I. I. Grekova, Vol. 145, No. 10, 1990, pp. 
42-43.

[7] A. N. Barkun, M. Bardou, E. J. Kuipers, R. H. Hunt, M. Martel, P. Sinclair and International Consensus Upper Gastrointestinal Bleeding Conference Group, "International Consensus Recommendations on the Management of Patients with Nonvariceal Upper Gastrointestinal Bleeding,” Annals of Internal Medicine, Vol. 152, No. 2, 2010, pp. 101-113.

[8] G. A. Agrawal, P. T. Johnson and E. K. Fishman, "Splenic Artery Aneurysms and Pseudoaneurysms: Clinical
Distinctions and CT Appearances,” American Journal of Roentgenology, Vol. 188, No. 4, 2007, pp. 992-999. http://dx.doi.org/10.2214/AJR.06.0794

[9] D. C. Madoff, A. Denys, M. J. Wallace, R. Murthy, S. Gupta, E.P. Pillsbury, K. Ahrar, B. Bessoud and M. E. Hicks, "Splenic Arterial Interventions: Anatomy, Indications, Technical Considerations, and Potential Complications,” Radiographics, Vol. 25, Suppl. 1, 2005, pp. S191S211. http://dx.doi.org/10.1148/rg.25si055504 